\title{
Stand Out in Class: restructuring the classroom environment to reduce sedentary behaviour in 9-10-year- olds - study protocol for a pilot cluster randomised controlled trial
}

Stacy A. Clemes ${ }^{1,2^{*}}$ (D), Daniel D. Bingham³ ${ }^{3}$ Natalie Pearson', Yu-Ling Chen', Charlotte Edwardson ${ }^{2,4}$, Rosemary McEachan ${ }^{3}$, Keith Tolfrey ${ }^{1,2}$, Lorraine Cale ${ }^{1}$, Gerry Richardson ${ }^{5}$, Mike Fray ${ }^{6}$, Stephan Bandelow ${ }^{1}$, Nishal Bhupendra Jaicim7, Jo Salmon ${ }^{8}$, David Dunstan ${ }^{9,10}$ and Sally E. Barber ${ }^{3}$

\begin{abstract}
Background: Sedentary behaviour (sitting) is a highly prevalent negative health behaviour, with individuals of all ages exposed to environments that promote prolonged sitting. Excessive sedentary behaviour adversely affects health in children and adults. As sedentary behaviour tracks from childhood into adulthood, the reduction of sedentary time in young people is key for the prevention of chronic diseases that result from excessive sitting in later life. The sedentary school classroom represents an ideal setting for environmental change, through the provision of sit-stand desks. Whilst the use of sit-stand desks in classrooms demonstrates positive effects in some key outcomes, evidence is currently limited by small samples and/or short intervention durations, with few studies adopting randomised controlled trial (RCT) designs. This paper describes the protocol of a pilot cluster RCT of a sit-stand desk intervention in primary school classrooms.

Methods/Design: A two-arm pilot cluster RCT will be conducted in eight primary schools (four intervention, four control) with at least 120 year 5 children (aged 9-10 years). Sit-stand desks will replace six standard desks in the intervention classrooms. Teachers will be encouraged to ensure all pupils are exposed to the sit-stand desks for at least $1 \mathrm{~h} /$ day on average using a rotation system. Schools assigned to the control arm will continue with their usual practice, no environmental changes will be made to their classrooms. Measurements will be taken at baseline, before randomisation, and at the end of the schools' academic year. In this study, the primary outcomes of interest will be school and participant recruitment and attrition, acceptability of the intervention, and acceptability and compliance to the proposed outcome measures (including activPAL-measured school-time and school-day sitting, accelerometermeasured physical activity, adiposity, blood pressure, cognitive function, academic progress, engagement, and behaviour) for inclusion in a definitive trial. A full process evaluation and an exploratory economic evaluation will also be conducted to further inform a definitive trial.

(Continued on next page)
\end{abstract}

\footnotetext{
* Correspondence: S.A.Clemes@lboro.ac.uk

${ }^{1}$ National Centre for Sport and Exercise Medicine, School of Sport, Exercise

and Health Sciences, Loughborough University, Loughborough, UK

${ }^{2} \mathrm{NIHR}$ Leicester Biomedical Research Centre, Leicester, UK

Full list of author information is available at the end of the article
}

(c) The Author(s). 2018 Open Access This article is distributed under the terms of the Creative Commons Attribution 4.0 International License (http://creativecommons.org/licenses/by/4.0/), which permits unrestricted use, distribution, and reproduction in any medium, provided you give appropriate credit to the original author(s) and the source, provide a link to the Creative Commons license, and indicate if changes were made. The Creative Commons Public Domain Dedication waiver (http://creativecommons.org/publicdomain/zero/1.0/) applies to the data made available in this article, unless otherwise stated. 
(Continued from previous page)

Discussion: The primary output of this study will be acceptability data to inform the development of a definitive cluster RCT designed to examine the efficacy of this intervention on health- and education-related outcomes in UK primary school children.

Trial registration: ISRCTN12915848 (retrospectively registered, date registered 9 November 2016).

Keywords: Sitting, Standing, Children, Sit-to-stand desks, Schools, Children's health, Education

\section{Background}

Technological advances and changes to our environment and lifestyle have led to increased time being spent in sedentary behaviours [1]. Sedentary behaviour (sitting, lying, and reclining and expending $<1.5$ metabolic equivalents [METs] [2]) is ubiquitous in modern society, with individuals of all ages exposed to environments that promote prolonged periods of sitting. Whilst it is acknowledged that physical activity is beneficial to health, sedentary behaviour has also been shown to adversely affect health. For example, high levels of sedentary behaviour have been independently associated with type 2 diabetes, cardiovascular disease, cancer and premature mortality in adults [3, 4].

Sitting is the most prevalent behaviour exhibited during waking hours in children from the UK, accounting for over $65 \%$ of their waking time [5], with some children reportedly sitting for over $10 \mathrm{~h} /$ day (> 70\% of waking hours) [6]. Adverse associations between sedentary behaviour and cardio-metabolic health risk markers (obesity, blood pressure, cholesterol, insulin) have been reported in children [7, 8]. Given this and as sedentary behaviours track throughout childhood into adolescence and adulthood [9], the reduction of sitting time in young people is pertinent for the primary and secondary prevention of chronic diseases that result from excessive sitting in adulthood [3, 4]. Furthermore, with the emergence of an increased cardio-metabolic health risk profile being observed in the first decade of life in some ethnic groups in the UK (for example, higher levels of fasting insulin and triglycerides have been observed in 9-10-years-old British South Asian children in comparison to white British children) [10], may suggest that these individuals could be more vulnerable to the adverse health effects of excessive sitting time. The early reduction of sedentary behaviour in children in such higher-risk groups could be an important strategy for reducing ethnicity-related health inequalities later in life.

Children are increasingly exposed to environments and social norms that encourage prolonged sitting; for example, because the school classroom is equipped with standard desks and chairs, children are expected to sit throughout most lessons. To counter the detrimental effects of prolonged sitting on children's health [7], strategies are needed to reverse the trend of increasing levels of sedentary behaviour. A meta-analysis of interventions targeting reductions in children's sedentary behaviour reported an overall decrease of $18 \mathrm{~min} /$ day of sitting and reductions in body mass index (BMI) [11]. Whilst most studies were school-based, sedentary behaviour was targeted via behaviour change strategies within non-school settings (i.e. screen time at home). No included studies employed changes to the classroom environment. Classrooms are conducive to high volumes of sitting, with children typically sitting for longer during school hours than non-school hours [12]. Environmental change within the classroom is therefore an obvious means to target children's sitting time and may address health inequalities in that this environment is accessible to all children. One such strategy in which environmental change to the classroom can be achieved is through the introduction of sit-stand desks. Unlike traditional classroom desks, sit-stand desks are height adjustable and enable the user to alternate between sitting and standing postures.

Rapid increases in sedentary time have been observed in children aged 11 years and above relative to younger age groups $[13,14]$. This trial will target year 5 classrooms, comprising children just below this age (910-year-olds), with the goal of reducing the typical rise in sedentary time seen during the transition into adolescence, given sedentary behaviours track from childhood into adolescence and adulthood [9]. Furthermore, evidence suggests that children in year 5 upwards are active participants in their own learning, making them an optimal target for classroom interventions that may facilitate learning [15].

Early-phase studies have demonstrated the feasibility of incorporating sit-stand desks in primary school classrooms over the short term (<12 weeks) $[6,16,17]$. We have previously observed that sit-stand desks enable pupils (9-10-year-olds) to alternate between sitting and standing, without disruption to teaching, learning or behaviour [6]. International studies have shown sit-stand desks in school classrooms to be effective in increasing energy expenditure $[18,19]$ and standing and movement [20] during the school day. Other research has shown that sit-stand desks in classrooms lead to improvements in children's posture and musculoskeletal comfort [21, 22] and levels of academic engagement [23] and 
achievement [22]. Two studies have assessed the impact on sitting directly, both revealed reductions in children's daily sitting time of approximately $1 \mathrm{~h}$ /day over 4 and 9 weeks follow-up [17, 24]. Recent reviews have concluded that the use of sit-stand desks in school classrooms shows promise; however, the evidence to date is limited to relatively small-scale studies with short intervention periods, and a lack of randomised controlled trials (RCTs) [25, 26]. Furthermore, limited research has examined the impact of this type of intervention on children's learning and academic achievement $[25,26]$. This pilot RCT will build on earlier research by assessing the longer-term acceptability of this low burden environmental intervention in primary schools in the UK, along with the acceptability of a range of health and education-related outcome measures to inform a definitive trial.

\section{Study aim and objectives}

The aim of this study is to undertake a pilot cluster RCT of the introduction of sit-stand desks in primary school classrooms to inform a future fully-powered definitive trial. A definitive trial would examine the impact of the introduction of sit-stand desks over one school year on weekday sitting time (the primary outcome), health and education related outcomes, with sub-group analyses for ethnicity. To inform such a trial, key information needs to be established around school and participant recruitment, acceptability of the intervention and outcome measures, and attrition rates. The objectives of this pilot study are therefore to

1. Establish and refine a recruitment strategy for schools and pupils.

2. Determine attrition in the trial (schools and children).

3. Determine completion rates for outcome measures (and whether these are sufficiently high to provide accurate data in a full trial).

4. Assess whether there are any differences in trial recruitment, retention and acceptability between ethnic groups.

5. Assess the acceptability of randomisation to schools to the intervention or standard practice.

6. Assess the acceptability of measurement instruments to teachers, children and parents, including the activPAL inclinometer as the tool for the measurement of the primary outcome.

7. Assess the acceptability of the intervention to teachers, children and parents.

8. Monitor any adverse effects, such as musculoskeletal discomfort and/or disruption to the classroom environment/teaching and learning to inform the design of a full trial and minimise or eliminate any such effects.

9. Assess intervention fidelity.

10. Derive preliminary estimates of the effect of the intervention on children's total daily sitting time, physical activity, indicators of health (markers of adiposity and blood pressure), cognitive function, quality of life, and academic performance, engagement and behaviour.

11. Estimate the standard deviation of the primary outcome to inform a sample size calculation for a full RCT.

12. Determine availability and completeness of economic data and conduct a preliminary assessment of potential cost-effectiveness.

\section{Methods/Design}

The design of this study is based on guidance from the UK Medical Research Council for developing and evaluating complex interventions [27]. This is a school-based, pilot two-armed cluster RCT with economic and process evaluations. Individuals (year 5 children, aged 9-10 years) will be the unit of analysis and schools (clusters) randomly assigned to one of two conditions: (1) six manually adjustable sit-stand desks incorporated into the classroom environment (intervention condition), or (2) current practice (control condition). Baseline measurements will precede randomisation. The sit-stand desks will be installed into the intervention classrooms following randomisation. An identical set of outcome measurements will be taken from all participants approximately 6 months after the baseline measures, when participants are at the end of year 5. Observations, interviews and focus groups with teachers, children and parents will be conducted throughout the intervention period as part of a full process evaluation. Figure 1 shows the study flow diagram, and Fig. 2 indicates the schedule of enrolment, intervention and outcome measurements. The project has been granted ethical approval by the Loughborough University Ethical Advisory Committee (reference R16-P027).

\section{Setting}

This study will be conducted in primary schools in Bradford. Bradford was chosen as the study location given its ethnic composition (predominantly South Asian and White British) and high levels of deprivation, health inequalities and childhood morbidity [28]. In 2016-17, $38 \%$ of year 6 children in Bradford were overweight or obese according to the National Child Measurement Programme [29]. Half of all babies born in Bradford are of South Asian origin and $60 \%$ are born into the poorest $20 \%$ of the population [28], with elevated risk factors for chronic diseases seen in South Asian children in comparison to White children $[10,30]$. The setting of this study therefore is fundamental in addressing the important issue of health inequalities, in that the intervention will be accessible to all children. In addition, the location enables us to pilot this intervention under challenging circumstances meaning that if it proves acceptable it is likely to be transferable to most schools. 


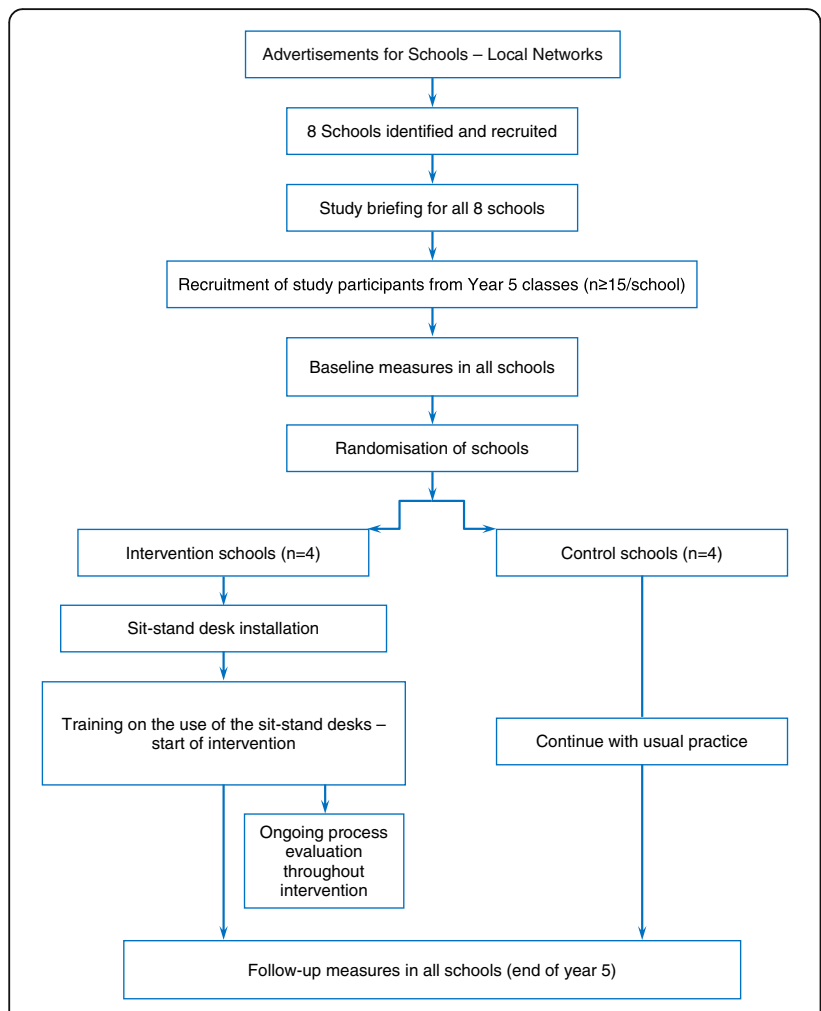

Fig. 1 Flow diagram for the Stand Out in Class pilot RCT

\section{Sample size}

Eight primary schools, each with at least 15 child participants (approximately $50 \%$ of a typical class) will be recruited giving a minimum total sample of 120 . This sample matches the minimum of four clusters per arm recommended for a cluster RCT [31], and the minimum sample size exceeds the recommendation for pilot trials [32]. Consequently, the sample will be sufficiently large to provide clear estimates of recruitment and follow-up for a definitive trial.

\section{School recruitment and inclusion criteria}

Government funded primary schools located in the City of Bradford will be invited to participate in the study following ethical approval. Private and designated special schools and schools with less than 25 pupils in year 5 will be excluded. Schools will also not be eligible for participation if they are running a sitting time reduction programme, or if they have a unique characteristic that prevents comparison with control schools. The study will be publicised to primary schools through existing local networks (e.g. Bradford Primary Improvement Partnership and Bradford's Public Forum for Education). We will aim to recruit four schools with predominantly South Asian pupils (>50\%) and four schools with predominantly White British pupils (>50\%). Interested schools will be followed-up via telephone and visits by the research team. Consent will be sought from school management (Head Teacher, Senior Teachers, Governors) and year 5 teachers to participate in the study. During recruitment, schools will be informed they may be randomised to a current practice control condition where they will be asked to maintain their usual classroom practice.

\section{Participant recruitment and inclusion criteria}

All year 5 children (9-10-years-old) within the participating schools will be eligible to participate. Parents will be sent a detailed information sheet about the study so that they can make an informed decision about their child's participation. They will also be invited to a school meeting outlining the study. Parental/guardian consent (through an opt-in form for their child to participate in the intervention evaluation) will be collected. Children will be asked to provide verbal assent at each data collection time point for the evaluation measures. Children without parental consent for their participation in the evaluation, or those who do not give their assent to participate in the evaluation, will be excluded from the evaluation measures described below. These children will however be able to use the sit-stand desks within their classrooms. Any children in the intervention schools with known contraindications (for example, a musculoskeletal injury, a wheelchair user) that would preclude periods of standing will be invited to participate in the evaluation measures and encouraged to use the sit-stand desk in a seated posture for inclusivity. These individuals will however be excluded from the analyses.

\section{Allocation to treatment groups}

To assess the acceptability of the intervention and proposed outcome measures for use in a definitive trial across an ethnically diverse sample, recruited schools will be stratified based on the ethnic composition of their pupils (identified above). Schools within each stratum will be randomised into the two study arms (intervention and control, using an allocation ratio of 1:1) by an independent statistician at the Leicester Clinical Trials Unit (CTU) following the completion of baseline measurements. Two schools with predominantly South Asian pupils and two schools with predominantly White British pupils will be randomised into the intervention and control arms (four schools in each arm). Stratification of schools will enable us to examine whether there are ethnic differences in terms of participant recruitment and retention, adherence to the outcome measures, and preliminary effects of the intervention on outcome measures. The statistician performing the analyses will be blinded to the schools allocation to the study arms, as will the community researchers undertaking the outcome measurements. 


\begin{tabular}{|c|c|c|c|c|c|c|}
\hline \multirow[b]{2}{*}{ TIMEPOINT } & \multirow{2}{*}{$\begin{array}{c}\text { Enrolment } \\
-t_{1}\end{array}$} & \multirow{2}{*}{$\begin{array}{l}\text { Baseline } \\
\text { Month } 0\end{array}$} & \multirow{2}{*}{$\begin{array}{c}\text { Allocation } \\
0\end{array}$} & \multicolumn{2}{|c|}{ Post-allocation } & \multirow{2}{*}{$\frac{\text { Close-out }}{t_{x}}$} \\
\hline & & & & $\begin{array}{c}\text { Start of } \\
\text { intervention } \\
\text { (month 1) } \\
\end{array}$ & $\begin{array}{c}\text { End of } \\
\text { intervention } \\
\text { (month 4) }\end{array}$ & \\
\hline \multicolumn{7}{|l|}{ ENROLMENT: } \\
\hline \multirow{5}{*}{$\begin{array}{r}\text { School recruitment } \\
\text { Eligibility screen } \\
\text { Parental Informed consent } \\
\text { Child assent } \\
\text { Allocation }\end{array}$} & $x$ & & & & & \\
\hline & & $x$ & & & & \\
\hline & & $x$ & & & & \\
\hline & & $x$ & & & & \\
\hline & & & $x$ & & & \\
\hline \multicolumn{7}{|l|}{ INTERVENTIONS: } \\
\hline \\
\hline \multicolumn{7}{|l|}{$\begin{array}{r}\text { Control group - usual } \\
\text { practice } \\
\end{array}$} \\
\hline \multicolumn{7}{|l|}{ ASSESSMENTS: } \\
\hline \multirow{2}{*}{$\begin{array}{r}\text { Feasibility outcomes } \\
\text { (recruitment) } \\
\text { Feasibility outcomes } \\
\text { (retention, compliance) }\end{array}$} & & $x$ & & & & \\
\hline & & & & & $x$ & $x$ \\
\hline \multirow{3}{*}{$\begin{array}{r}\text { Participant demographics } \\
\text { Objectively measured } \\
\text { sitting/standing } \\
\text { Objectively measured } \\
\text { physical activity }\end{array}$} & & $x$ & & & & \\
\hline & & $x$ & & & $x$ & \\
\hline & & $x$ & & & $x$ & \\
\hline Stature and body mass & & $x$ & & & $x$ & \\
\hline \multirow{2}{*}{$\begin{array}{r}\text { BMI and body composition } \\
\text { Blood pressure }\end{array}$} & & $x$ & & & $x$ & \\
\hline & & $x$ & & & $x$ & \\
\hline \multirow{4}{*}{$\begin{array}{r}\text { Cognitive function } \\
\text { Pupil behavior (teacher } \\
\text { reported) } \\
\text { Academic attainment } \\
\text { (School reported) } \\
\text { Learning engagement and } \\
\text { disaffection }\end{array}$} & & $x$ & & & $x$ & \\
\hline & & $x$ & & & $x$ & \\
\hline & & & & & & $x$ \\
\hline & & $x$ & & & $x$ & \\
\hline \multirow{5}{*}{$\begin{array}{r}\text { Postural comfort } \\
\text { Health-related quality of life } \\
\text { Health and education } \\
\text { resource use } \\
\text { Process evaluation: } \\
\text { interviews/focus groups } \\
\text { Process evaluation: } \\
\text { classroom observations }\end{array}$} & & $x$ & & & $x$ & \\
\hline & & $x$ & & & $x$ & \\
\hline & & $x$ & & & $x$ & \\
\hline & & & & $x$ & $x$ & $x$ \\
\hline & & & & $x$ & $x$ & \\
\hline
\end{tabular}

Fig. 2 Standard Protocol Items: Recommendations for Interventional Trials (SPIRIT) diagram illustrating the design and timescales of the pilot Stand Out in Class RCT

\section{Experimental intervention}

Six sit-stand desks will be placed in one year 5 classroom (replacing standard desks) in each intervention school for two school terms. The research team will support teachers in the development of a rotation plan to ensure that all children in their class are exposed to the sit-stand desks for at least $1 \mathrm{~h} /$ day on average across the week. Stools or chairs will remain and children will be free to choose whether they sit or stand. Teachers and pupils in the intervention classrooms will receive training on sit-stand desk use by the research team. Teachers will also receive a Professional Development Manual containing information on the health benefits of reducing prolonged sitting and information on correct posture when standing at the desks. The manual and training will focus on encouraging correct adoption of the intervention targeting key barriers and facilitators to sit-stand desk use, identified from our previous work $[6,33]$ and from the Capability, Opportunity, and Motivation to perform a Behaviour (COM-B) model within the Behaviour Change Wheel [34] and the Theoretical Domains Framework [35] (e.g. self-efficacy, motivation and knowledge). Standardised behaviour change techniques (e.g. goal setting, instruction) [36] will also be used. A summary of the intervention components is shown in Table 1 and Fig. 3 represents a simple logic model for the Stand Out in Class intervention. Additional file 1 details potential intervention barriers, solutions and hypothesised mediating processes informed by the above theoretical frameworks. 
Table 1 Components of the Stand Out in Class intervention

\begin{tabular}{|c|c|c|c|}
\hline Intervention component & Target domain & Meditating variable & Description \\
\hline Adjustable sit-stand desks & Environment & Exposure to desks & $\begin{array}{l}\text { Six adjustable sit-stand desks introduced into } \\
\text { the classroom }\end{array}$ \\
\hline Nudging prompts & Environment & $\begin{array}{l}\text { Children choose to stand rather } \\
\text { than sit when using desks }\end{array}$ & $\begin{array}{l}\text { Stickers placed upon each of the sit-stand desks } \\
\text { - Have you stood up this lesson? } \\
\text { - Standing tall and proud this lesson? }\end{array}$ \\
\hline 2-h one-to-one meeting & Teacher & Exposure to desks & $\begin{array}{l}\text { 2-h meeting with teacher which will cover: } \\
\text { - Why it is important to increase standing/reduce } \\
\text { sitting } \\
\text { - Importance of exposure to sit-stand desks } \\
\text { - Safety-how to use the desks } \\
\text { - Rotation plan and example, plus creation of a } \\
\text { rotation plan for the following } 2 \text { weeks }\end{array}$ \\
\hline Professional Development Manual & Teacher & Exposure to desks & $\begin{array}{l}\text { Cover topics such as } \\
\text { - Why it is important to increase standing/reduce } \\
\text { sitting } \\
\text { - Importance of exposure to sit-stand desks } \\
\text { - Safety-how to use the desks } \\
\text { - Rotation plan example }\end{array}$ \\
\hline Planned weekly rotation plan & Teacher & Exposure to desks & $\begin{array}{l}\text { Teacher creates a predetermined rotation plan } \\
\text { and keeps a record of whether this was met or } \\
\text { not—-simple tick sheet }\end{array}$ \\
\hline Fortnightly support with practitioners & Teacher & Exposure to desks & $\begin{array}{l}\text { Phone or face-to-face meeting with researchers/ } \\
\text { practitioners-discuss any issues around } \\
\text { implementation of rotation plans. }\end{array}$ \\
\hline 30-min workshop & Children & $\begin{array}{l}\text { Exposure to desks } \\
\text { Children choose to stand rather } \\
\text { than sit when using desks }\end{array}$ & $\begin{array}{l}\text { Cover topics such as } \\
\text { - Why it is important to increase standing/reduce } \\
\text { sitting } \\
\text { - Importance of exposure to sit-stand desks } \\
\text { - Safety-how to use the desks } \\
\text { - Rotation plan and how it will work } \\
\text { - Discuss other intervention aspects-social } \\
\text { contract and competition }\end{array}$ \\
\hline Standing champion/leader & Children & $\begin{array}{l}\text { Exposure to desks } \\
\text { Children choose to stand rather } \\
\text { than sit when using desks }\end{array}$ & $\begin{array}{l}\text { One child in a group is chosen as a standing } \\
\text { champion with responsibility of reminding the } \\
\text { teacher of the rotation plan. }\end{array}$ \\
\hline Group contract & Children & $\begin{array}{l}\text { Exposure to desks } \\
\text { Children choose to stand rather } \\
\text { than sit when using desks }\end{array}$ & $\begin{array}{l}\text { Children are all asked to sign a large contract } \\
\text { which will state: } \\
\text { I will try my best to } \\
\text { - Stand up in a good posture when using the } \\
\text { sit-stand desk. } \\
\text { - Not to disturb the class when I stand up at } \\
\text { my sit-stand desk. } \\
\text { - Remember when it is my turn to use the } \\
\text { sit-stand desk. } \\
\text { - Be very careful not to hurt myself or other } \\
\text { people when I use the sit-stand desk. }\end{array}$ \\
\hline
\end{tabular}

\section{Control arm}

To compare the effects of the intervention against usual practice (i.e. the provision of standard classroom desks), schools assigned to the control arm will be requested to continue with their usual practice and lesson delivery, no environmental changes will be made to their classrooms. The year 5 participants in the control schools will be asked to complete the same study measurements as those in the intervention schools at the same time points. Upon completion of the study, control schools will receive a report summarising their pupils' sitting and physical activity data. They will also receive adapted materials (i.e. the Professional Development Manual provided to teachers in the intervention schools outlining the benefits of reducing sitting but excluding references to sit-stand desks) upon completion of all follow-up evaluation measures.

\section{Measurements}

Trial feasibility-related outcomes

The predominant aim of this pilot study is to establish school and participant recruitment and retention rates, acceptability of the intervention and proposed outcome measures, intervention and measurement fidelity, and 


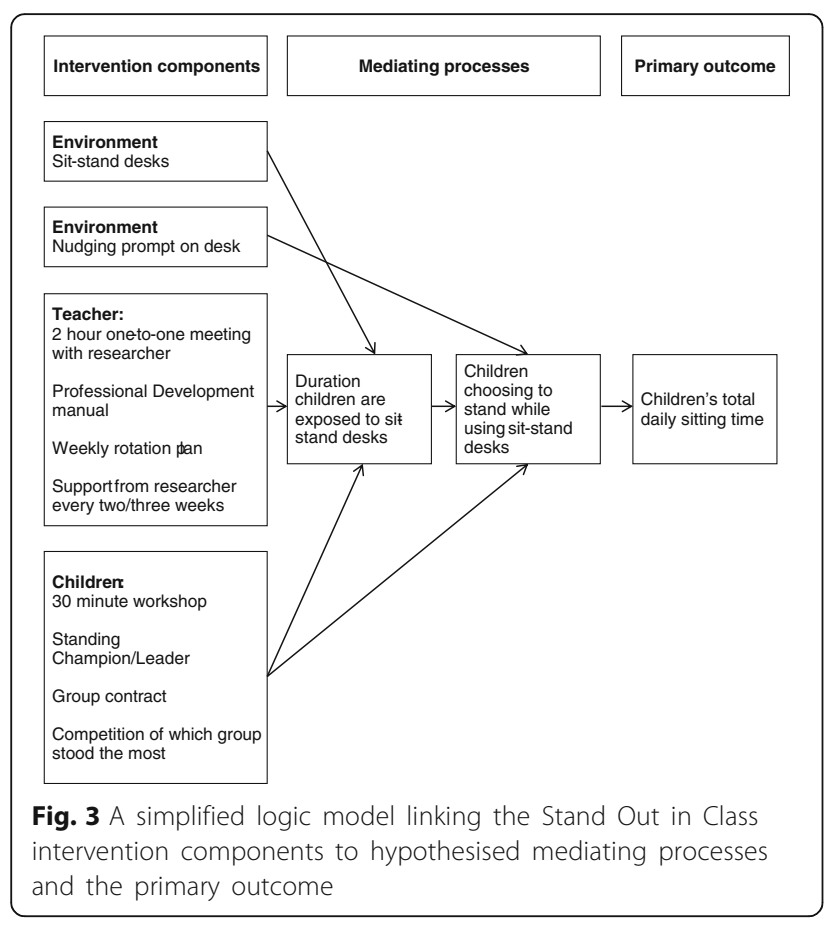

the availability and completeness of economic data for an estimation of potential cost-effectiveness in order to inform the development of a fully powered definitive trial. Study uptake will be monitored by recording the number of schools and pupils approached, and the number agreeing to participate (objective 1). Withdrawal rates of schools and children (objective 2) and completion rates for outcome measures will be summarised (objective 3), and these feasibility outcomes will be compared between ethnic groups (objective 4).

\section{Process evaluation}

A series of interviews and focus groups with teachers, children and parents will be conducted following the completion of baseline measures and randomisation to explore the acceptability of trial procedures, including randomisation (objective 5), and the acceptability of the measurement instruments (objective 6). Eight one-to-one interviews (one per teacher) will be undertaken with the participating year 5 teachers during this phase of the process evaluation, and eight separate focus groups (one per school) will be undertaken with children and parents. We will aim to recruit between four and six participants for each focus group conducted throughout the process evaluation. Acceptability of the intervention (objective 7), and children's, teacher's and parent's perceptions and experiences of the intervention and outcome measures (including any negative effects such as discomfort from the monitoring equipment, reasons for non-compliance to the outcome measures, or class disruption) will be obtained during the intervention through a further set of interviews $(n=4$, with teachers from the intervention schools) and focus groups (four with children and four with parents from the intervention schools) (objective 8). Differences in trial and intervention acceptability between ethnic groups will be explored as part of the analyses from the focus groups (objective 4). Towards the end of the intervention, four one-to-one interviews will be conducted (one per intervention school) with senior staff (head teachers/deputy head teachers) to further examine the acceptability of the intervention (objective 7). Table 2 provides a detailed plan of the measures and methodologies which will be used in the process evaluation.

To assess intervention, fidelity intervention classrooms will be observed by a member of the research team for a duration of at least half a school day during the Spring and Summer terms. These classroom observations will take place once per school term (Spring and Summer) for each intervention school (eight observations in total, two per intervention school). During these observations, every $10 \mathrm{~min}$ the researcher will record the number of boys and girls who are sitting or standing when using the sit-stand desks. Field notes will also be taken to document the occurrence of any intervention components (i.e. use of prompt cards, engagement with a standing champion, see Table 1) during the observation period. Children's posture during sit-stand desk use will also be recorded using a postural analysis recording system based on the Portable Ergonomic Observation (PEO) [21] to assess any future risk of musculoskeletal injury (objectives 8 and 9).

\section{Health- and education-related outcome measures}

All health-related outcome measurements will be taken twice, at baseline prior to randomisation, and approximately 6 months after baseline when pupils are at the end of year 5 . The likely primary outcome in a definitive trial would be change in average daily school-day sitting time. Sitting will be measured objectively for seven consecutive days during each measurement period using the activPAL3 micro accelerometer. The activPAL3 will be waterproofed (using a nitrile sleeve and hypoallergenic Hypafix [BSN Medical] dressing) and participants will be requested to wear the device continuously ( $24 \mathrm{~h}$ /day) on the anterior aspect of their right thigh during each measurement period. Participants will be provided with a brief diary during each monitoring period where they will be requested to document time in bed and any periods of non-wear.

The use of the activPAL to objectively measure sitting has increased in recent years [37], and the device has been successfully used as a primary outcome measure in previous school-based sedentary behaviour interventions $[6,17,24]$ and is recommended for use in interventions 
Table 2 The Stand Out in Class pilot RCT process evaluation plan

\begin{tabular}{lll}
\hline Areas to measure & General process questions & $\begin{array}{l}\text { Data source and data collection } \\
\text { method }\end{array}$ \\
\hline $\begin{array}{ll}\text { Acceptability of randomisation } \\
\text { and measurement tools } \\
\text { (objectives } 5 \text { and 6) }\end{array}$ & $\begin{array}{l}\text { How did schools feel about } \\
\text { being randomised to }\end{array}$ & $\begin{array}{l}\text { Interviews with teachers } \\
\text { In-class focus groups with }\end{array}$ \\
& intervention/control arms? & children \\
& How did schools/children/parents & Focus groups with parents \\
& outcome assessments? & Recruitment data (numbers \\
& Did schools/children/parents find & consenting), and missing data \\
& outcome assessments acceptable? & One-to-one interviews with \\
& What were the reasons for not & parents and children not \\
& participating in the trial and/or & participating and/or not \\
not complying to the outcome & complying
\end{tabular}

Intervention acceptability and fidelity (objectives 7 and 9)

\section{Was the intervention implemented} as planned?
Interviews with intervention teachers

Observations of lessons

Teachers logs and telephone interviews with intervention teachers

Focus groups with children (in class)

Interviews with teachers

Focus groups with parents children/parents with sit-stand desks?

How did schools/children/parents perceive the outcomes and usefulness of sit-stand desks
Intervention acceptability and fidelity - strategies to facilitate implementation (objective 7 and 9)

Intervention acceptability and fidelity - quality of delivery (objectives 7 and 9)
What strategies were used to support introduction of standing desks?

How were these strategies perceived by staff involved within project?

How well were sit-stand desks introduced?

What is the quality of the sit-stand desks and professional manual?
Interviews with teachers

Interviews with teachers Focus groups with children (in class)
Total numbers and sampling strategy

Eight teachers from participating schools (end of summer term) Eight focus groups with children (one per school) after baseline assessments.

Eight focus groups with parents (one per school) after baseline assessments.

Data collected at baseline and follow-up Interviews conducted after baseline assessments

Four intervention teachers Time-points (T):

T1: After desk installation

T2: End of year 5

Four intervention schools observed once per term

Four intervention teachers recording use of desks in log book and brief telephone contact every 2 weeks

Four in class focus groups with children from intervention schools: Time points:

T1: After desk installation

T2: End of year 5

Four intervention teachers

T1: After desk installation

T2: End of year 5

Four parent focus groups from intervention schools (after desk installation)

Four intervention teachers Time-points:

T1: After desk installation

T2: End of year 5

Four intervention teachers

Time-points:

T1: After desk installation

T2: End of year 5

4 in class focus groups with children from intervention schools:

T1: After desk installation

T2: End of year 5

Interviews with teachers and head teachers
Four intervention teachers (end of year 5)

Four interviews with head teachers from intervention schools (end of year 5) when the primary outcome measure is sitting [38]. It is regarded as the most accurate method of assessing sitting behaviour in free-living settings [39] and has been shown to be almost $100 \%$ accurate in measuring sitting, standing, walking and postural transitions in children [40, 41]. Whilst total daily school-day sitting time is likely to be the primary outcome for a future trial, we will also extract classroom and leisure-time sitting, standing and stepping time, along with the number of transitions between sitting and standing from the activPAL data. Periods of non-wear and sleep time will be excluded from the analyses using an automated algorithm [42], supplemented with cross-checking against participant's diary entries. We will examine any positive (i.e. reductions in sitting) or compensatory effects (increases in sitting) of the intervention on children's behaviour out of school hours. The variability (standard deviation) of the data from the proposed primary outcome will be 
used to inform a sample size calculation for a definitive trial (objective 11).

Proposed secondary outcomes for a definitive trial include objectively measured physical activity. While the activPAL provides a valid measure of posture, it has not been well validated for assessment of various physical activity intensities among children. Therefore, children will wear the ActiGraph GT3X+ accelerometer on the waist continuously ( $24 \mathrm{~h} /$ day) for seven consecutive days, concurrently with the activPAL, during each measurement period. Periods of non-wear will be documented in a brief diary provided with the devices. Waist worn accelerometers have traditionally been considered the criterion measure of children's physical activity [43]. The ActiGraph is the most commonly used accelerometer in field-based research and has been shown to have acceptable reliability and validity in paediatric populations [44]. Times spent in light (26-573 counts per $15 \mathrm{~s}$ epoch) and moderate-to-vigorous intensity ( $\geq 574$ counts per $15 \mathrm{~s}$ epoch) activity throughout the day, and during and out of school hours will be extracted from the ActiGraph data using the Evenson cut-points [45]. In a comparative study examining the classification accuracy of five different ActiGraph cut-points for determining children's and adolescent's physical activity intensity, against indirect calorimetry, the Evenson cut-points were highlighted as the most accurate across all intensity levels and are therefore recommended for use in this age group [46]. Periods of non-wear and sleep time will be excluded from the analyses during the processing of the ActiGraph data, supplemented with cross-checking against participant's diary entries.

At each measurement point, children's stature and body mass (both assessed without shoes) will be measured directly using standard procedures by trained research staff and BMI will be calculated and converted to a BMI percentile based on UK reference data [47]. Body composition (percentage body fat and fat mass) will be assessed via bio-impedance analysis, using Tanita DC-360S body composition scales which contain specific algorithms for children. Blood pressure will be measured from the left arm after at least a 5-min period of quiet sitting using a semi-automated recorder (Omron HEM-907) with a paediatric cuff, in accordance with current recommendations [48]. Three measurements of blood pressure will be taken; each measurement will be separated by a 2 -min rest period. The mean systolic and diastolic blood pressures recorded from the second and third assessments will be calculated and used in the analyses.

A set of objective cognitive function tests will also be administered via a validated software package. The software will be installed on school computers enabling a group of students (those with parental consent to participate in the intervention evaluation) to undertake these assessments at the same time in the classroom under the supervision of two researchers. Participants will undertake a practise run through of the cognitive function test battery a day before the test day. The cognitive function test battery will take children approximately $15 \mathrm{~min}$ to complete and will include the Corsi block tapping test [49], the Stroop test [50] and the rapid visual information processing (RVIP) task [51]. The Corsi block tapping test is a measure of visuo-spatial working memory capacity [49]. Performance on the Corsi block tapping test is linearly associated with age in typically developing children [52], and the original version has exhibited a test-retest reliability coefficient of 0.7 (Pearson's correlation coefficient) in 11-year-olds [53]. The Stroop test measures sensitivity to interference and the ability to suppress an automated response (reading colour names in favour of naming the font colour); it is a commonly used measure of selective attention and executive function [54]. The complex reaction time measure within the computerised Stroop test has been shown to exhibit a test-retest reliability of 0.55 (Pearson's correlation coefficient) in a combined sample of children and adults [55]. The RVIP is a measure of sustained attention and has been shown to exhibit an internal reliability coefficient (assessed using Cronbach's alpha) of 0.49 in school-age children [56].

Measures of pupils' academic progress and attainment will be collected using routine assessment data collected by the schools at half-termly intervals. The impact of the intervention on pupil's behaviour will be assessed using the Strengths and Difficulties questionnaire completed by teachers at baseline and follow-up. This questionnaire, when completed by teachers, has been shown to provide a valid indicator of children's behaviour (convergent validity: Pearson's correlation coefficient with the Rutter questionnaire $=0.92$ [57]). Children will also report their engagement and disaffection with their own learning [58] (correlation coefficients between pupil and teacher reports of the components of engagement using this measure range from 0.26 to 0.44 [59]) and their postural comfort [21]. In addition, the Paediatric Quality of Life Inventory (PEDS-QL) [60] and EuroQol 5-dimension Youth (EQ-5D-Y) [61] will be completed by children to provide a measure of self-reported quality of life at each measurement point. The construct validity of the PEDS-QL has been previously demonstrated with healthy children displaying significantly higher scores on this measure in comparison to acutely or chronically ill children [60]. Responses on the EQ-5D-Y have been shown to correlate with other measures of children's health-related quality of life (convergent validity correlation coefficients up to 0.56 [62]). To further inform the economic analysis (objective 12), teachers and parents 
will also complete a questionnaire (created for the purpose of this study) assessing participants' health- and education-related resource use at baseline and follow-up. Basic demographic information (sex, age, ethnicity and postcode to determine Index of Multiple Deprivation (IMD) as an indicator of socio-economic status) will be collected at baseline.

\section{School and participant appreciation}

As a thank you for participating in the pilot trial, all schools (intervention and control) will receive a donation of $£ 200$ at the end of the trial. A $£ 5$ gift voucher will be given to children following the completion of both baseline and follow-up measures to encourage a timely return of the accelerometers.

\section{Economic analysis}

The availability and completeness of economic data will be established as part of this pilot study (objective 12). Resource-use information will be collected, which will include the cost of the sit-stand desks, along with participants' health- (e.g. General Practitioner visits) and education-related resource use (e.g. requirements for additional tutoring). Proposed outcomes within a definitive trial will be based on two sectors, health and education. For the former we will use the PEDS-QL [60] and EQ-5D-Y [61] delivered at baseline and follow-up to assess children's health-related quality of life; for the latter, we will separately present measures of academic performance obtained through the schools' routine assessment data. A preliminary cost-effectiveness analysis will be conducted to inform the value of a full trial and to make recommendations for the design of the full trial. We will present cost and PEDS-QL and EQ-5D-Y data as an incremental cost-effectiveness ratio where the additional cost associated with the provision of sit-stand desks is formally compared with the additional benefit of providing sit-stand desks. We will also present a comparison of pupils' academic achievement in each group. A brief scoping review will be carried out to identify existing model(s) that link the shorter-term outcomes to longer-term effects (on both health and education outcomes [63]) and where feasible, we will use these model(s) to examine the likely additional costs and benefits of the intervention over the longer term.

\section{Data analysis}

\section{Statistical analysis}

This study will be analysed and reported according to the Consolidation Standards of Reporting Trials (CONSORT) statement for cluster RCTs [64]. Data will be analysed on a complete-case basis. The purpose of the primary analysis is to assess the feasibility of recruitment and adherence/retention of primary schools and pupils to a sit-stand desk intervention. As this is a pilot trial, the primary analyses will mainly utilise descriptive statistics. We will summarise the number of schools approached, the number agreeing to participate, the proportion of children within each school with parental consent to participate in the study evaluation, the number of children completing the study protocol, retention rates, and the number providing valid outcome measurement data at baseline and follow-up. The study acceptability data will be presented for the sample as a whole and stratified according to study arm (intervention and control) and ethnicity (South Asian and White British).

While the main aim of this study is to establish acceptability, feasibility, recruitment rates and sample size to inform a definitive trial, and although effectiveness will unlikely be established with the small sample size, we will examine the primary and secondary outcomes to mimic practice for a full trial. Results from this analysis will be treated as preliminary and interpreted with caution $[65,66]$. As the number of clusters is low, cluster summary statistics will be used rather than multi-level modelling $[67,68]$. The analysis will be carried out using children as the unit of analysis with change in average total school-day sitting time as the primary outcome. A weighted linear regression model will be used to compare the intervention arms weighted by the number of participants followed up in each cluster and adjusted for baseline total daily sitting time on school days for each cluster. To examine preliminary effects of the intervention on the secondary outcomes, the same analytical approach will be adopted as for the primary outcome (objective 10). These analyses will provide preliminary evidence of the effectiveness of the intervention and will in-part inform the decision of whether a definitive trial should be undertaken.

Sensitivity analyses will be performed on the proposed primary outcome for a full trial of average weekday sitting time, and on the secondary activPAL (standing and stepping time) and ActiGraph (time in light physical activity and moderate-to-vigorous physical activity) variables. This will be achieved by including pupils who have worn the activPAL and ActiGraph (with a minimum valid wear time of $8 \mathrm{~h}$ each day) for at least 1, 2, 3 and 4 weekdays, and on at least 2 weekdays and 1 weekend day at both baseline and follow-up.

The objective of this pilot study is to estimate the standard deviation of the primary outcome to inform a full trial. The intraclass correlation coefficient (ICC) to inform the sample size calculation of the definitive trial will be estimated from published literature. The ICC will not be estimated from this pilot study, because multilevel modelling is considered to be an unsuitable analysis method in the present study due to low number of 
clusters and an ICC estimate from such a model would not be sufficiently robust to inform a sample size calculation for a definitive trial.

\section{Qualitative analyses}

Audio-recordings of interviews and focus groups with teachers, parents and children collected as part of the process evaluation will be transcribed verbatim and analysed using framework analysis [69, 70], using the Normalisation Process Theory [71] and the Theoretical Domains Framework [35] as the underpinning theoretical framework. These will be supplemented with field notes from observation sessions. Collectively, these will provide information on the acceptability of the trial procedures including randomisation, the measurement instruments and the overall acceptability of the intervention.

\section{Data management and research governance}

Anonymised data will be entered into a secure and validated clinical data management system provided by the Leicester CTU; this database (InferMed Macro v4) includes a series of quality control mechanisms to ensure that the data collected are complete and accurate. The study will be sponsored by Loughborough University. Two groups will be created to oversee the study; an independent Trial Steering Committee (TSC) and a Project Committee. As the study is regarded as low risk, the TSC will take on the role of a Data Monitoring Committee and review any serious adverse events which are thought to be intervention related and monitor progress with data collection. The TSC will meet every 6 months and include the principle investigators (Clemes and Barber), an independent chair, two independent external members (including a statistician) and two school representatives. The Project Committee will comprise the principle investigators, all co-investigators and those concerned with the day to day running of the study. The Project Committee will meet monthly and provide an update report for the TSC. Additional file 2 details the Standard Protocol Items: Recommendations for Interventional Trials (SPIRIT) checklist for this protocol paper.

\section{Discussion}

The pathogenesis of chronic diseases such as type 2 diabetes and cardiovascular disease have origins in childhood [72]. The precursors of chronic disease risk in young people are a major public health issue, given links between lifestyle behaviours and disease risk. Sedentary behaviour is highly prevalent in children and adversely associated with adiposity/weight gain and clustered metabolic risk [7, 73]. As sedentary behaviours track from childhood into adulthood [9], reducing children's sitting time could reduce the risk of numerous chronic conditions linked to prolonged sitting in adulthood, including obesity, some cancers, type 2 diabetes and cardiovascular disease mortality $[3,4,74]$.

The need for more, and more effective, interventions targeting the primary prevention of chronic diseases by encouraging healthy lifestyles in children has been explicitly acknowledged. The Chief Medical Officer for England's 2012 Annual Report, 'Our Children Deserve Better: Prevention Pays' and the UK's 'Childhood Obesity Plan for Action' highlight the importance of the school environment in promoting healthy behaviours $[75,76]$. While there are examples of school-based sedentary behaviour interventions from Australia [77], New Zealand $[17,24]$ and the USA $[19,23]$, differences in school systems, curricula and cultures preclude direct translation of these findings to the UK. Schools are a crucial setting for addressing health inequalities, with classroom-based interventions being accessible to all children. The ethnically diverse and socio-economically deprived geographical location of this study allows us to test this type of intervention under challenging circumstances. If acceptable, it is likely to be transferable to most UK schools.

This pilot trial will provide the detailed information and insight needed to design a definitive cluster RCT (if preliminary estimates of effectiveness are observed), which will examine the impact of sit-stand desks in the classroom environment on health- and education-related outcomes in UK primary school children.

\section{Additional files}

Additional file 1: Potential intervention domain barriers and solutions to barriers using the Capability, Opportunity, Motivation to perform a Behaviour model (COM-B), Theoretical Domains Framework (TDF) and behaviour change techniques (BCT). (DOCX $45 \mathrm{~kb}$ )

Additional file 2: SPIRIT checklist. (DOCX $51 \mathrm{~kb}$ )

\section{Abbreviations}

BMl: Body mass index; COM-B model: Capability, Opportunity, and Motivation to perform a Behaviour model; CONSORT: Consolidation Standards of Reporting Trials; CTU: Clinical trials unit; EQ-5D-Y: EuroQol 5-dimension Youth; ICC: Intraclass correlation coefficient; IMD: Index of Multiple Deprivation; METs: Metabolic equivalents; PEDS-QL: Paediatric Quality of Life Inventory; PEO: Portable Ergonomic Observation; RCT: Randomised controlled trial; RVIP: Rapid visual information processing task; SPIRIT: Standard Protocol Items: Recommendations for Interventional Trials; TSC: Trial Steering Committee; UK: United Kingdom

\section{Acknowledgements}

This study was primarily funded by a grant received from the NIHR Public Health Research Programme (reference NIHR PHR 14/231/20). SC, CE and KT are supported by the NIHR Leicester Biomedical Research Centre-Lifestyle theme. SEB, RMC, DB and GR are supported by the Healthy Children, Healthy Families Theme of the NIHR CLAHRC Yorkshire and Humber. This study was designed and delivered in collaboration with the Leicester Clinical Trials Unit (CTU), a UKCRC Registered Clinical Trials Unit in receipt of NIHR CTU support funding. None of the study funders have had involvement in the Trial 
Steering Committee, the data analysis, data interpretation, data collection or writing of the paper. The views expressed in this publication are those of the authors and not necessarily those of the funding bodies listed. Loughborough University is the sponsor for this trial.

\section{Funding}

This study is funded by the NIHR Public Health Research Programme (reference NIHR PHR 14/231/20) and supported by the NIHR Leicester Biomedical Research Centre-Lifestyle theme. The views expressed are those of the authors and not necessarily those of the NHS, the NIHR or the Department of Health and Social Care.

\section{Availability of data and materials}

The datasets generated during the study will be available from the corresponding author on reasonable request

\section{Authors' contributions}

All authors have made substantial contributions to the concept and design of the study. The study was conceived by SC, SEB, NP, DB, CE, KT, LC, RMCE, $\mathrm{SB}, \mathrm{DD}, \mathrm{JS}$ and MF. NP and YLC are the qualified Research Associates, and DB is the local study coordinator. GR is the health economics analyst, and NBJ is the trial statistician. The first draft of this manuscript was written by SC with input from all other authors. All authors have edited and critically reviewed the paper for intellectual content and approved the final version of the paper.

\section{Ethics approval and consent to participate}

The study has received ethical approval from the Loughborough University Ethical Advisory Committee (reference R16-P027). Parents/guardians have provided written informed consent for their child to participate in the evaluation of the intervention.

\section{Competing interests}

The sit-stand desks used in this study are being supplied via an in-kind donation from Ergotron Inc., USA. The company has played no role in the study design and they will not play any role in the data analyses or in the preparation of the final outcome papers. There are no further competing interests to declare.

\section{Publisher's Note}

Springer Nature remains neutral with regard to jurisdictional claims in published maps and institutional affiliations.

\section{Author details}

${ }^{1}$ National Centre for Sport and Exercise Medicine, School of Sport, Exercise and Health Sciences, Loughborough University, Loughborough, UK. ${ }^{2} \mathrm{NIHR}$ Leicester Biomedical Research Centre, Leicester, UK. ${ }^{3}$ Bradford Institute for Health Research, Bradford Teaching Hospitals Foundation Trust, Bradford, UK. ${ }^{4}$ Diabetes Research Centre, University of Leicester, Leicester, UK. ${ }^{5}$ Centre for Health Economics, University of York, Heslington, York, UK. 'Loughborough Design School, Loughborough University, Loughborough, UK. ${ }^{7}$ Leicester Clinical Trials Unit, University of Leicester, Leicester, UK. ${ }^{8}$ Institute for Physical Activity and Nutrition, School of Exercise and Nutrition Sciences, Deakin University, Melbourne, Australia. ${ }^{9}$ Baker IDI Heart and Diabetes Institute, Melbourne, Australia. ${ }^{10}$ Mary Mackillop Institute for Health Research, Australian Catholic University, Melbourne, Australia.

\section{Received: 19 January 2018 Accepted: 10 May 2018}

\section{Published online: 24 May 2018}

\section{References}

1. Salmon J, Tremblay MS, Marshall SJ, Hume C. Health risks, correlates, and interventions to reduce sedentary behavior in young people. Am J Prev Med. 2011;41(2):197-206.

2. Tremblay MS, Aubert S, Barnes JD, Saunders TJ, Carson V, LatimerCheung AE, Chastin SFM, Altenburg TM, Chinapaw MJM, Participants STCP. Sedentary behavior research network (SBRN)-terminology consensus project process and outcome. Int J Behav Nutr Phys Act. 2017;14(1):75

3. Wilmot EG, Edwardson CL, Achana FA, Davies MJ, Gorely T, Gray LJ, Khunti K, Yates T, Biddle SJ. Sedentary time in adults and the association with diabetes, cardiovascular disease and death: systematic review and metaanalysis. Diabetologia. 2012;55(11):2895-905.

4. Biswas A, Oh PI, Faulkner GE, Bajaj RR, Silver MA, Mitchell MS, Alter DA. Sedentary time and its association with risk for disease incidence, mortality, and hospitalization in adults: a systematic review and meta-analysis. Ann Intern Med. 2015;162(2):123-32.

5. Steele RM, van Sluijs EM, Cassidy A, Griffin SJ, Ekelund U. Targeting sedentary time or moderate- and vigorous-intensity activity: independent relations with adiposity in a population-based sample of 10-y-old British children. Am J Clin Nutr. 2009;90(5):1185-92.

6. Clemes SA, Barber SE, Bingham DD, Ridgers ND, Fletcher E, Pearson N, Salmon J, Dunstan DW. Reducing children's classroom sitting time using sit-to-stand desks: findings from pilot studies in UK and Australian primary schools. J Public Health (Oxf). 2016;38(3):526-33.

7. Tremblay MS, LeBlanc AG, Kho ME, Saunders TJ, Larouche R, Colley RC, Goldfield G, Connor Gorber S. Systematic review of sedentary behaviour and health indicators in school-aged children and youth. Int J Behav Nutr Phys Act. 2011:8:98.

8. Marshall SJ, Biddle SJ, Gorely T, Cameron N, Murdey I. Relationships between media use, body fatness and physical activity in children and youth: a meta-analysis. Int J Obes Relat Metab Disord. 2004;28(10):1238-46.

9. Biddle SJ, Pearson N, Ross GM, Braithwaite R. Tracking of sedentary behaviours of young people: a systematic review. Prev Med. 2010;51(5): 345-51.

10. Whincup PH, Nightingale CM, Owen CG, Rudnicka AR, Gibb I, McKay CM, Donin AS, Sattar N, Alberti KG, Cook DG. Early emergence of ethnic differences in type 2 diabetes precursors in the UK: the child heart and health study in England (CHASE study). PLoS Med. 2010;7(4): e1000263.

11. van Grieken A, Ezendam NP, Paulis WD, van der Wouden JC, Raat H. Primary prevention of overweight in children and adolescents: a metaanalysis of the effectiveness of interventions aiming to decrease sedentary behaviour. Int J Behav Nutr Phys Act. 2012;9:61.

12. Abbott RA, Straker LM, Mathiassen SE. Patterning of children's sedentary time at and away from school. Obesity 2013;21(1):E131-E133.

13. Craig R, Mindell J, Hirani V. Health Survey for England 2008: Volume 1, Physical activity and fitness. The Health and Social Care Information Centre; 2009.

14. Pearson N, Haycraft E, P Johnston J, Atkin AJ. Sedentary behaviour across the primary-secondary school transition: a systematic review. Prev Med 2017:94(40-47.

15. Ginsburg H, Opper S. Piaget's theory of intellectual development third edition. Englewood Cliffs: Prentice-Hall, Inc; 1988.

16. Blake JJ, Benden ME, Wendel ML. Using stand/sit workstations in classrooms: lessons learned from a pilot study in Texas. J Public Health Manag Pract. 2012;18(5):412-5.

17. Hinckson EA, Aminian S, Ikeda E, Stewart T, Oliver M, Duncan S, Schofield G. Acceptability of standing workstations in elementary schools: a pilot study. Prev Med. 2013;56(1):82-5.

18. Benden ME, Blake JJ, Wendel ML, Huber JC Jr. The impact of stand-biased desks in classrooms on calorie expenditure in children. Am J Public Health. 2011;101(8):1433-6.

19. Benden ME, Zhao H, Jeffrey CE, Wendel ML, Blake JJ. The evaluation of the impact of a stand-biased desk on energy expenditure and physical activity for elementary school students. Int J Environ Res Public Health. 2014;11(9):9361-75.

20. Lanningham-Foster L, Foster RC, McCrady SK, Manohar CU, Jensen TB, Mitre NG, Hill JO, Levine JA. Changing the school environment to increase physical activity in children. Obesity. 2008;16(8):1849-53.

21. Benden ME, Pickens A, Shipp E, Perry J, Schneider D. Evaluating a school based childhood obesity intervention for posture and comfort. Health. 2013;5(8A3):54-60.

22. Koskelo R, Vuorikari K, Hanninen O. Sitting and standing postures are corrected by adjustable furniture with lowered muscle tension in highschool students. Ergonomics. 2007;50(10):1643-56.

23. Dornhecker M, Blake JJ, Benden ME, Zhao H, Wendel ML. The effect of stand-biased desks on academic engagement: an exploratory study. Int J Health Promot Educ. 2015;53(5):271-80.

24. Aminian S, Hinckson EA, Stewart T. Modifying the classroom environment to increase standing and reduce sitting. Building Research \& Information. 2015;43(5):631-45.

25. Sherry AP, Pearson N, Clemes SA. The effects of standing desks within the school classroom: a systematic review. Prev Med Rep. 2016;3:338-47. 
26. Hinckson E, Salmon J, Benden M, Clemes SA, Sudholz B, Barber SE, Aminian S, Ridgers ND. Standing classrooms: research and lessons learned from around the world. Sports Med. 2016;46(7):977-87.

27. Craig P, Dieppe P, Macintyre S, Michie S, Nazareth I, Petticrew M. Developing and evaluating complex interventions: the new Medical Research Council guidance. Int J Nurs Stud. 2013;50(5):587-92.

28. Wright J, Small N, Raynor P, Tuffnell D, Bhopal R, Cameron N, Fairley L, Lawlor DA, Parslow R, Petherick ES, et al. Cohort profile: the born in Bradford multiethnic family cohort study. Int J Epidemiol. 2013;42(4):978-91.

29. National Statistics. National Child Measurement Programme, England, 2016/17 school year. NHS Digital: Health and Social Care Information Centre; 2017.

30. Whincup PH, Gilg JA, Papacosta O, Seymour C, Miller GJ, Alberti KG, Cook DG. Early evidence of ethnic differences in cardiovascular risk: cross sectional comparison of British south Asian and white children. BMJ. 2002:324(7338):635.

31. Murray D. Design and analysis of group-randomized trials. Oxford: Oxford University Press; 1998.

32. Julious SA. Sample size of 12 per group rule of thumb for a pilot study. Pharm Stat. 2005;4(4):287-91.

33. Sherry AP, Pearson N, Ridgers ND, Barber SE, Bingham DD, Nagy LC, Dunstan D, Clemes S: The effectiveness of sit-to-stand desks to reduce sitting time within a primary school classroom: an 8 month controlled trial. In the proceedings of the International Society of Behavioral Nutrition and Physical Activity (ISBNPA) Annual Meeting: 2017; Victoria, Canada.

34. Michie S, van Stralen MM, West R. The behaviour change wheel: a new method for characterising and designing behaviour change interventions. Implement Sci. 2011;6:42.

35. Michie S, Johnston M, Abraham C, Lawton R, Parker D, Walker A, Psychological Theory G. Making psychological theory useful for implementing evidence based practice: a consensus approach. Qual Saf Health Care. 2005;14(1):26-33.

36. Michie S, Richardson M, Johnston M, Abraham C, Francis J, Hardeman W, Eccles MP, Cane J, Wood CE. The behavior change technique taxonomy (v1) of 93 hierarchically clustered techniques: building an international consensus for the reporting of behavior change interventions. Ann Behav Med. 2013;46(1):81-95.

37. Edwardson C, Winkler E, Bodicoat DH, Yates T, Davies M, Dunstan D, Healy GN. Considerations when using the activPAL monitor in field-based research with adult populations. J Sport Health Sci. 2017;6:162-78.

38. Kozey-Keadle S, Libertine A, Lyden K, Staudenmayer J, Freedson PS. Validation of wearable monitors for assessing sedentary behavior. Med Sci Sports Exerc. 2011;43(8):1561-7.

39. Atkin AJ, Gorely T, Clemes SA, Yates T, Edwardson C, Brage S, Salmon J, Marshall SJ, Biddle SJ. Methods of measurement in epidemiology: sedentary behaviour. Int J Epidemiol. 2012;41(5):1460-71.

40. Aminian S, Hinckson EA. Examining the validity of the ActivPAL monitor in measuring posture and ambulatory movement in children. Int J Behav Nutr Phys Act. 2012;9:119.

41. Davies G, Reilly JJ, McGowan AJ, Dall PM, Granat MH, Validity PJY. Practical utility, and reliability of the activPAL in preschool children. Med Sci Sports Exerc. 2012;44(4):761-8.

42. Winkler EA, Bodicoat DH, Healy GN, Bakrania K, Yates T, Owen N, Dunstan DW, Edwardson CL. Identifying adults' valid waking wear time by automated estimation in activPAL data collected with a $24 \mathrm{~h}$ wear protocol. Physiol Meas. 2016;37(10):1653-68.

43. Dollman J, Okely AD, Hardy L, Timperio A, Salmon J, Hills APA. hitchhiker's guide to assessing young people's physical activity: deciding what method to use. J Sci Med Sport. 2009;12(5):518-25.

44. Trost SG, Ward DS, Moorehead SM, Watson PD, Riner W, Burke JR. Validity of the computer science and applications (CSA) activity monitor in children. Med Sci Sports Exerc. 1998;30(4):629-33

45. Evenson KR, Catellier DJ, Gill K, Ondrak KS, McMurray RG. Calibration of two objective measures of physical activity for children. J Sports Sci. 2008;26(14): 1557-65.

46. Trost SG, Loprinzi PD, Moore R, Pfeiffer KA. Comparison of accelerometer cut points for predicting activity intensity in youth. Med Sci Sports Exerc. 2011;43(7):1360-8.

47. Cole TJ, Freeman JV, Preece MA. Body mass index reference curves for the UK, 1990. Arch Dis Child. 1995;73(1):25-9.

48. O'Brien E, Asmar R, Beilin L, Imai Y, Mallion JM, Mancia G, Mengden T, Myers M, Padfield P, Palatini P, et al. European Society of Hypertension recommendations for conventional, ambulatory and home blood pressure measurement. J Hypertens. 2003;21(5):821-48.

49. Corsi PM. Human memory and the medial temporal region of the brain. Diss Abstr Int. 1973;34(2-B):891.

50. MacLeod CM. Half a century of research on the Stroop effect: an integrative review. Psychol Bull. 1991;109(2):163-203.

51. Bakan P. Extraversion-introversion and improvement in an auditory vigilance task. Br J Psychol. 1959;50:325-32.

52. Farrell Pagulayan K, Busch RM, Medina KL, Bartok JA, Krikorian R. Developmental normative data for the Corsi block-tapping task. J Clin Exp Neuropsychol. 2006;28(6):1043-52.

53. Orsini A. Corsi's block-tapping test: standardization and concurrent validity with WISC-R for children aged 11 to 16. Percept Mot Skills. 1994;79(3 Pt 2): 1547-54.

54. Cooper SB, Bandelow S, Nute ML, Dring KJ, Stannard RL, Morris JG, Nevill ME. Sprint-based exercise and cognitive function in adolescents. Prev Med Rep. 2016;4:155-61.

55. Gualtieri $C$, Johnson LG. Reliability and validity of a computerized neurocognitive test battery, CNS vital signs. Arch Clin Neuropsychol. 2006;21(7):623-43.

56. Syvaoja HJ, Tammelin TH, Ahonen T, Kankaanpaa A, Kantomaa MT. The associations of objectively measured physical activity and sedentary time with cognitive functions in school-aged children. PLoS One. 2014;9(7): e103559.

57. Goodman R. The strengths and difficulties questionnaire: a research note. J Child Psychol Psychiatry. 1997;38(5):581-6.

58. Skinner EA, Marchand G, Furrer C, Kindermann T. Engagement and disaffection in the classroom: part of a larger motivational dynamic. J Educ Psychol. 2008;100(4):765-81.

59. Skinner EA, Kindermann T, Furrer C. A motivational perspective on engagement and disaffection. Educ Psychol Meas. 2009;69(3):493-525.

60. Varni JW, Seid M, Kurtin PS. PedsQL 4.0: reliability and validity of the pediatric quality of life inventory version 4.0 generic core scales in healthy and patient populations. Med Care. 2001;39(8):800-12.

61. Wille N, Badia X, Bonsel G, Burstrom K, Cavrini G, Devlin N, Egmar AC, Greiner W, Gusi N, Herdman M, et al. Development of the EQ-5D-Y: a childfriendly version of the EQ-5D. Qual Life Res. 2010;19(6):875-86.

62. Ravens-Sieberer U, Wille N, Badia X, Bonsel G, Burstrom K, Cavrini G, Devlin N, Egmar AC, Gusi N, Herdman M, et al. Feasibility, reliability, and validity of the EQ-5D-Y: results from a multinational study. Qual Life Res. 2010;19(6):887-97.

63. Heckman JJ. The economics, technology, and neuroscience of human capability formation. Proc Natl Acad Sci U S A. 2007;104(33):13250-5.

64. Campbell MK, Piaggio G, Elbourne DR, Altman DG, Group C. Consort 2010 statement: extension to cluster randomised trials. BMJ. 2012;345:e5661.

65. Lancaster GA, Dodd S, Williamson PR. Design and analysis of pilot studies: recommendations for good practice. J Eval Clin Pract. 2004;10(2):307-12

66. Arain M, Campbell MJ, Cooper CL, Lancaster GA. What is a pilot or feasibility study? A review of current practice and editorial policy. BMC Med Res Methodol. 2010;10:67.

67. Bland JM, Kerry SM. Statistics notes. Trials randomised in clusters. BMJ. 1997; 315(7108):600

68. Kerry SM, Bland JM. Analysis of a trial randomised in clusters. BMJ. 1998; 316(7124):54.

69. Ritchie J, Spencer L. Qualitative data analysis for applied policy research. In: Bryman, Burgess, editors. Analysing Qualitative Data. London: Routledge; 1994. p. 173-94.

70. Gale NK, Heath G, Cameron E, Rashid S, Redwood S. Using the framework method for the analysis of qualitative data in multi-disciplinary health research. BMC Med Res Methodol. 2013;13:117.

71. May $C$ et al. Normalization Process Theory On-line Users' Manual and Toolkit. Available from http://www.normalizationprocess.org/. Accessed 11 Mar 2015.

72. Expert Panel on Integrated Guidelines for Cardiovascular Health and Risk Reduction in Children and Adolescents. Expert panel on integrated guidelines for cardiovascular health and risk reduction in children and adolescents: summary report. Pediatrics. 2011;128(Suppl 5):S213-56.

73. Saunders TJ, Chaput JP, Tremblay MS. Sedentary behaviour as an emerging risk factor for cardiometabolic diseases in children and youth. Can J Diabetes. 2014;38(1):53-61.

74. Hu FB, Li TY, Colditz GA, Willett WC, Manson JE. Television watching and other sedentary behaviors in relation to risk of obesity and type 2 diabetes mellitus in women. JAMA. 2003;289(14):1785-91. 
75. Annual Report of the Chief Medical Officer 2012. Our children deserve better: prevention pays. London: Crown Copyright; 2013.

76. Department of Health. Childhood obesity: a plan for action. London: Crown Copyright; 2016.

77. Salmon J, Arundell L, Hume C, Brown H, Hesketh K, Dunstan DW, Daly RM, Pearson N, Cerin E, Moodie M, et al. A cluster-randomized controlled trial to reduce sedentary behavior and promote physical activity and health of 8-9 year olds: the transform-us! study. BMC Public Health. 2011;11:759.

Ready to submit your research? Choose BMC and benefit from:

- fast, convenient online submission

- thorough peer review by experienced researchers in your field

- rapid publication on acceptance

- support for research data, including large and complex data types

- gold Open Access which fosters wider collaboration and increased citations

- maximum visibility for your research: over $100 \mathrm{M}$ website views per year 